\title{
FICOFLORA DE AMBIENTES LENTICOS - ESTUDO PRELIMINAR DA REGIÃO DE CHARQUEADAS, RIO GRANDE DO SUL, BRASIL, COM VISTAS À AVALIAÇÃO AMBIENTAL ${ }^{1}$
}

\author{
Zulanira Meyer Rosa ${ }^{2}$ \\ Ieti Ungaretti ${ }^{3}$ \\ Lisete Maria Kremer ${ }^{3}$ \\ Sandra Maria Alves da Silva ${ }^{4}$ \\ Vera Lúcia Maróstica Callegaro ${ }^{2}$ \\ Vera Regina Werner ${ }^{3}$
}

Recebido em 23.04.1987. Aceito em 29.10.1987.

RESUMO - São apresentados os resultados do estudo do metafíton, bentos e de comunidades fitoplanctônicas ocorrentes em ambientes lênticos de Charqueadas e municípios vizinhos, regiâo esta sujeita à influência de mineração e processamento de carvão mineral. $\mathrm{O}$ estudo baseou-se em 46 amostras coletadas em julho e agosto de 1986. Dos 213 táxons específicos e infra-específicos identificados, 95 constituem-se em primeiro registro de ocorrência no Estado. Os grupos melhor representados quanto ao número de espécies ocorrentes foram Chlorophyta (Desmídias) e Bacillariophyta (Diatomáceas). Foi constatada presença de espécies indicadas na literatura como organismos de ambientes ácidos, com maior representatividade no bentos e metafíton de biótopos sujeitos à influência direta de rejeito de carvăo. Nesses sistemas ácidos, foi registrada no fitoplâncton dominância de organismos nanoplanctônicos da classe Chrysophyceae, estando quantitativamente bem representadas, no bentos, as Euglenophyta, Bacillariophyta e Chlorophyta. A estrutura das comunidades fitoplanctônicas mostrou baixos índices de diversidade, caracterizando um desequilibrio na composição específica das mesmas.

Palavras-chave: Fitoplâncton/Metafíton/Bentos/Sistemas lênticos ácidos/Rio Grande do Sul.

\footnotetext{
${ }^{1}$ Trabalho realizado no Museu de Ciências Naturais da Fundação Zoobotânica do Rio Grande do Sul, financiado pelo acordo FUNDATEC-ELETROSUL para elaboração do RIMA DA U.T. Jacuí I. Apresentado no XXXVIII Congresso Nacional de Botânica.

${ }^{2}$ Pesquisador do Museu de Ciências Naturais da Fundação Zoobotânica do Rio Grande do Sul (MCN/FZB) - Av. Salvador França, 1427, CEP 90610 - Porto Alegre, RS; bolsistas do Conselho Nacional de Desenvolvimento Cientifico e Tecnológico (CNPq).

${ }^{3}$ Bolsistas do Conselho Nacional de Desenvolvimento Científico e Tecnológico (CNPq) no MCN/FZB.

${ }^{4}$ Pesquisadora do MCN/FZB.
} 


\begin{abstract}
Metaphyton, benthos and phytoplantonic communities in lentic systems in the region of Charqueadas Rio Grande do Sul, Brazil.) Metaphyton, benthos and phytoplanktonic communities occurring in lentic systems were studied in July and August 1986. The study was based on 46 samples collected in Charqueadas and other neighboring areas in the State of Rio Grande do Sul, a region which suffers the effects of coal mining and processing. Out of the 213 specific and infraspecific taxa identified, 95 are a first record in the State of Rio Grande do Sul. As far as the number of occurring species is concerned, the best represented groups were Chlorophyta (Desmids) and Bacillariophyta (Diatoms). Organisms indicative of acid systems were found, mostly in benthos and metaphyton environments subject to the influence of coal wastes. In these acid systems, phytoplankton showed nanoplanktonic organisms of the group Chrysophyceae; Euglenophyta, Bacillariophyta and Chlorophyta were all well represented in the metaphyton and benthos. The structure of the phytoplanktonic communites showed low diversity, which suggests an unbalanced composition.
\end{abstract}

Key words: Phytoplankton/Metaphyton/Benthos/Acid lentic systems/Rio Grande do Sul State.

\title{
Introdução
}

Estudos da estrutura de comunidades de algas associados ao conhecimento de condições físicas e químicas da água podem constituir-se em bons indicadores de impacto ambiental oriundo de despejos industriais e domésticos. A diversidade específica das comunidades pode retratar a estabilidade do sistema, sendo que índices de diversidade baixos refletem, em geral, condições extremas de poluição ou pureza das águas (MARGALEF, 1974; ROUND, 1981).

O conhecimento taxonômico da ficoflora é de relevância para que se possa avaliar, de forma mais precisa, condições ambientais específicas.

Até o presente momento, para os ambientes lênticos de Charqueadas e municípios limítrofes, inexistem publicações referentes a algas, restringindo-se o conhecimento da ficoflora da região a relatórios técnicos, comunicaçōes apresentadas em congressos e trabalhos científicos ainda em fase de publicação.

Este trabalho teve por objetivo realizar um inventário preliminar da ficoflora de Charqueadas e adjacências e efetuar estudos da estrutura de comunidades do fitoplâncton na tentativa de avaliar as condições ambientais presentes. Constitui-se em parte do Relatório de Impacto do Meio Ambiente (RIMA) realizado com vistas à implantação da Usina Termelétrica Jacuí I.

\section{Material e métodos}

Foram obtidas 46 amostras de material fitoplanctônico, metafítico e bentônico de ambientes lênticos localizados nos municípios de Charqueadas, Triunfo, São Jerônimo, Arroio dos Ratos e Guaíba. Os corpos d'água amostrados são açudes, alagados e sacos junto ao Rio Jacuí.

O critério utilizado para seleção dos ambientes estudados baseou-se na direção que tomam os ventos na região, que é predominantemente Sudeste, de acordo com "Observações meteorológicas do Instituto de Pesquisas Agronômicas - IPAGRO - da Secretaria da Agricultura, RIO GRANDE DO SUL (1968-1985)", segundo médias anuais obtidas para a região de Guaíba. Com 
base nesses dados, foram escolhidos, principalmente, açudes localizados nos quadrantes Sudeste e Sudoeste, para tornar possível a evidenciação de quaisquer alterações que pudessem estar sendo provocadas por poluentes aéreos industriais oriundos, principalmente, da queima de carvão mineral por usina termelétrica e indústria siderúrgica sediadas em Charqueadas.

Como segundo critério, foram selecionados, além dos ambientes que se encontravam aparentemente o mais próximo das condições naturais, também aqueles que se constituiam em depósitos de cinza de carvão mineral, sendo que, somente para estes últimos, foi estudada a comunidade bentônica. $\mathrm{Na}$ avaliação dos sistemas amostrados foram observadas também as características peculiares de cada ambiente, tendo sido caracterizados cinco tipos básicos de ambientes lênticos na região, por análise comparativa entre os mesmos.

Para o estudo taxonômico propriamente dito, foram obtidas amostras de material fitoplanctônico através de concentração de material de superfície (com rede de plâncton de abertura de malha de 36 micrômetros), de material metafítico, através de espremido de macrófitos aquáticos (quando esses se fizeram representar, tendo sido os mesmos coletados para identificação) e de material bentônico, através de raspado de fundo ou da utilização de draga de Ekman. Estas amostras foram fixadas com TRANSEAU, na proporção 1:1.

Visando ao estudo da estrutura de comunidades fitoplanctônicas, foram obtidas amostras através de coleta de frasco, na superfície da água, sendo as mesmas fixadas com lugol-acético e mantidas sob refrigeração.

Para a maioria dos biótopos, foi realizada amostragem numa mesma data, sendo coletadas amostras de diferentes comunidades representadas e, objetivando a obtenção de dados complementares, foram repetidas as amostras nos ambientes $\mathrm{n}^{\circ} 5,6$ e 13. As expediçōes de coleta foram efetivadas nos dias $20,21,24$, 26 de julho e 9 de agosto de 1986.

As amostras encontram-se tombadas no Herbário Prof. Dr. Alarich R.H. Schultz (HAS) e estão registradas sob números: 16516 a 16519, 16523 a 16525 , 16531 a 16541,16543 a 16563,16565 a 16572 e 16577. A correspondência desses registros a seus respectivos ambientes e datas de coleta encontra-se junto à relação a seguir, sendo que os sistemas amostrados estão indicados no mapa $\mathrm{n}^{\circ} 1$.

$\mathrm{n}^{\circ} 1$ Açude da Fazenda Bianchini, Município de Guaíba, HAS16539-41 (24.VII.1986).

$n^{\circ} 2$ Açude no Horto Florestal da CEEE (talhão 45), Município de Guaíba, HAS16516 (20.VII.1986).

$n^{\circ} 3$ Açude no Horto Florestal da CEEE (talhão I), Município de Guaíba, HAS16523-25 (20.VII. 1986).

n. 4 Açude da Granja Carola, Município de Guaíba, HAS16531-32 (20.VII.1986).

n. 5 Açude no Município de Charqueadas - Passo Sete, HAS15533 (26.VII.1986); HAS16534 (20.VII.1986).

$\mathrm{n}^{\circ} 6$ Açude no Município de Charqueadas (constituído por deposição de cinza de carvão), HAS16535-37 (20.VII.1986); HAS16538 (26.VII.1986). 
MAPA 1 - Mapa indicativo dos pontos de amostragem, junto à região de Charqueadas e adjacências.

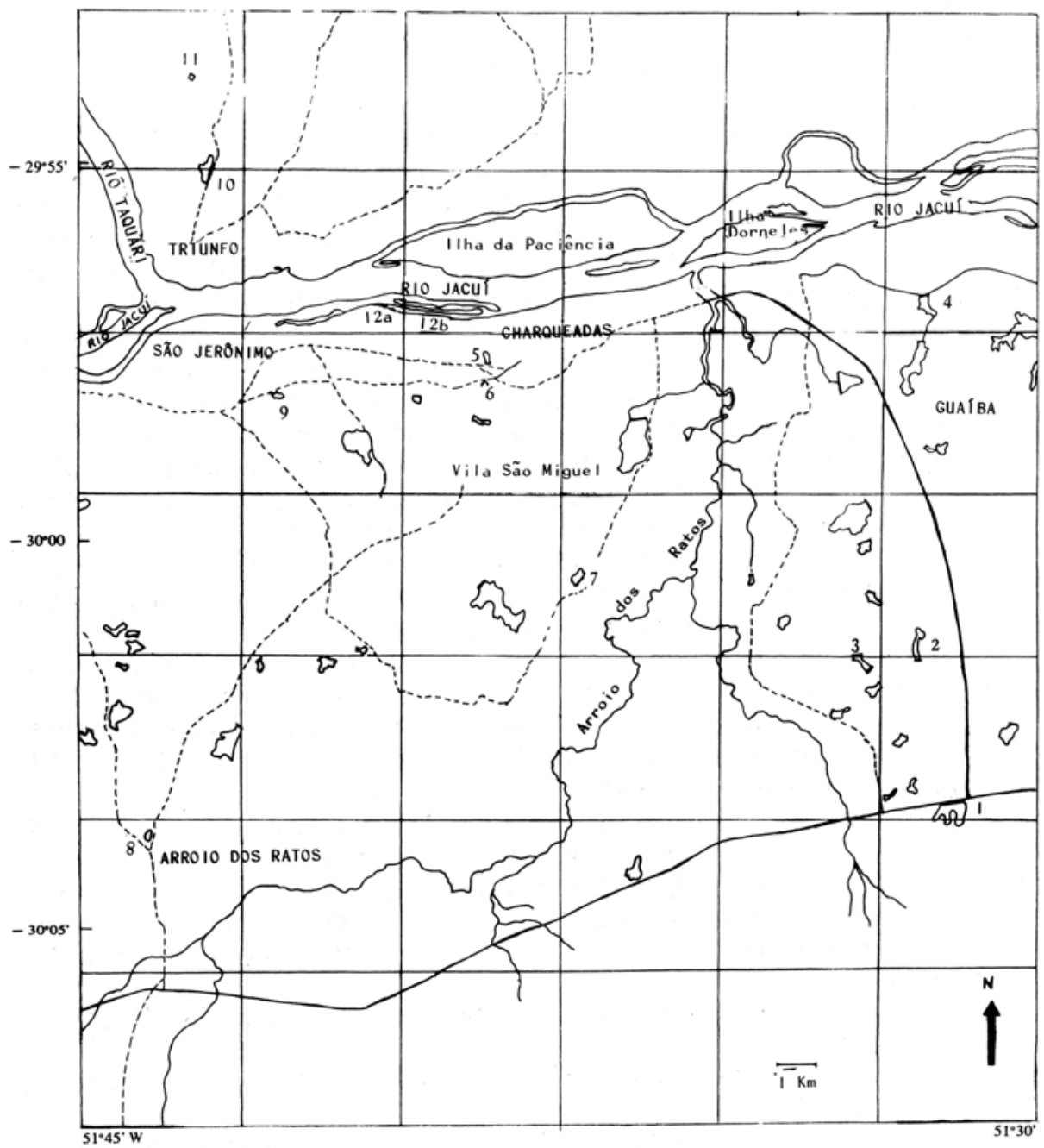

$\mathrm{n}^{\circ} 7$ Açude localizado no Município de Arroio dos Ratos, junto à Colônia Penal Agrícola, HAS16543-48 (26.VII.1986).

$\mathrm{n}^{\circ} 8$ Alagado no Município de Arroio dos Ratos, HAS16549-51 (26.VII.1986).

$n^{\circ} 9$ Açude Lago Parque Clube, Município de São Jerônimo, HAS16552-54 (26.VII.1986).

$\mathrm{n}^{\circ} 10$ Açude Boa Vista, Município de Triunfo, HAS16555-57 (26.VII.1986).

$\mathrm{n}^{\circ} 11$ Açude do Horto Florestal Renner, Município de Triunfo, HAS16558-61 (26.VII.1986). 
$n^{\circ} 12$ (a,b) Saco do Jacuí, na região limítrofe entre os municípios de Charqueadas e São Jerónimo; ponto à oeste do saco (12a) e ponto intermediário entre o limite oeste e o rio propriamente dito (12b), HAS16562-63, 16565-69 e 16577 (9.VIII.1986).

$n^{\circ} 13$ Saco da Illa dos Domeles, à oeste, HAS16570-71 (21.VII. 1986); HAS16572 (24.VII.1986).

Foram realizadas, em açudes localizados no quadrante sudeste e em saco do Rio Jacuí, mediçōes puntuais de alguns parámetros físicos e químicos, tais como: oxigennio dissolvido, com oxímetro YSI, modelo 57; transparéncia, com disco de Secchi; condutividade e temperatura, com Condutivimetro-salinómetro YSI, modelo 33; e pH, com medidor WTW, modelo D 8120 . WEIHEIM. Os dados abióticos foram obtidos para análise comparativa entre os diferentes ambientes estudados, entretanto, nảo foram efetuadas mediçōes em todos 08 ambientes pelo fato do equipamento não estar disponível em algumas das expediç̧̄es realizadas.

As análises taxonômicas foram efetuadas em microscópio de pesquisa Leitz, com utilização de lâminas permanentes e não-permanentes, com base em literatura especializada. Para o estudo de diatomáceas, foi efetuada oxidação do material segundo a técnica de MÜLLER-MELCHERS \& FERRANDO (1956), com adaptaçōes. A sistemática adotada para ordenação de grandes grupos segue ROUND (1973).

A análise de densidade e da estrutura de comunidades (espécies dominantes, espécies abundantes e diversidade específica) foi efetuada com base em amostras do fitoplâncton processadas em microscópio invertido Wild M 40, em câmaras de sedimentação (método de UTERMÖHL, 1958), sendo os resultados calculados em número de organismos $/ \mathrm{ml}$. O critério utilizado para determinação de espécies abundantes e dominantes foi o descrito em LOBO \& LEIGHTON (1986). Os índices de diversidade foram obtidos segundo SHANNON \& WEAVER (1949), com adaptação baseada em PIELOU (1975).

A tentativa de avaliação ambiental apoiou-se, principalmente, na análise da estrutura de comunidades do fitoplâncton; foram tambem considerados os resultados taxonômicos do metafíton e do bentos, com base em dados existentes na literatura (SCHOEMAN, 1973; SLÁDECEK, 1973; HUBER-PESTALOZZI, 1955; COESEL et alii, 1978; e outros) referentes à ecologia das espécies melhor representadas.

\section{Resultados}

Os ambientes lênticos selecionados para estudo, em Charqueadas e municipios limítrofes, foram caracterizados, de maneira geral, em cinco tipos basicos, sendo que os resultados de algumas mediçōes abióticas realizadas encontram-se relacionados na tabela $n^{\circ} 4$.

1. Açudes e alagados com escassa vegetação arbórea marginal (Eucalyptus * sp.) e vegetação rasteira formada basicamente por gramineas; cobertura de macrofitos inferior a $10 \%$ da superficie total do biotopo e pH moderadamente 


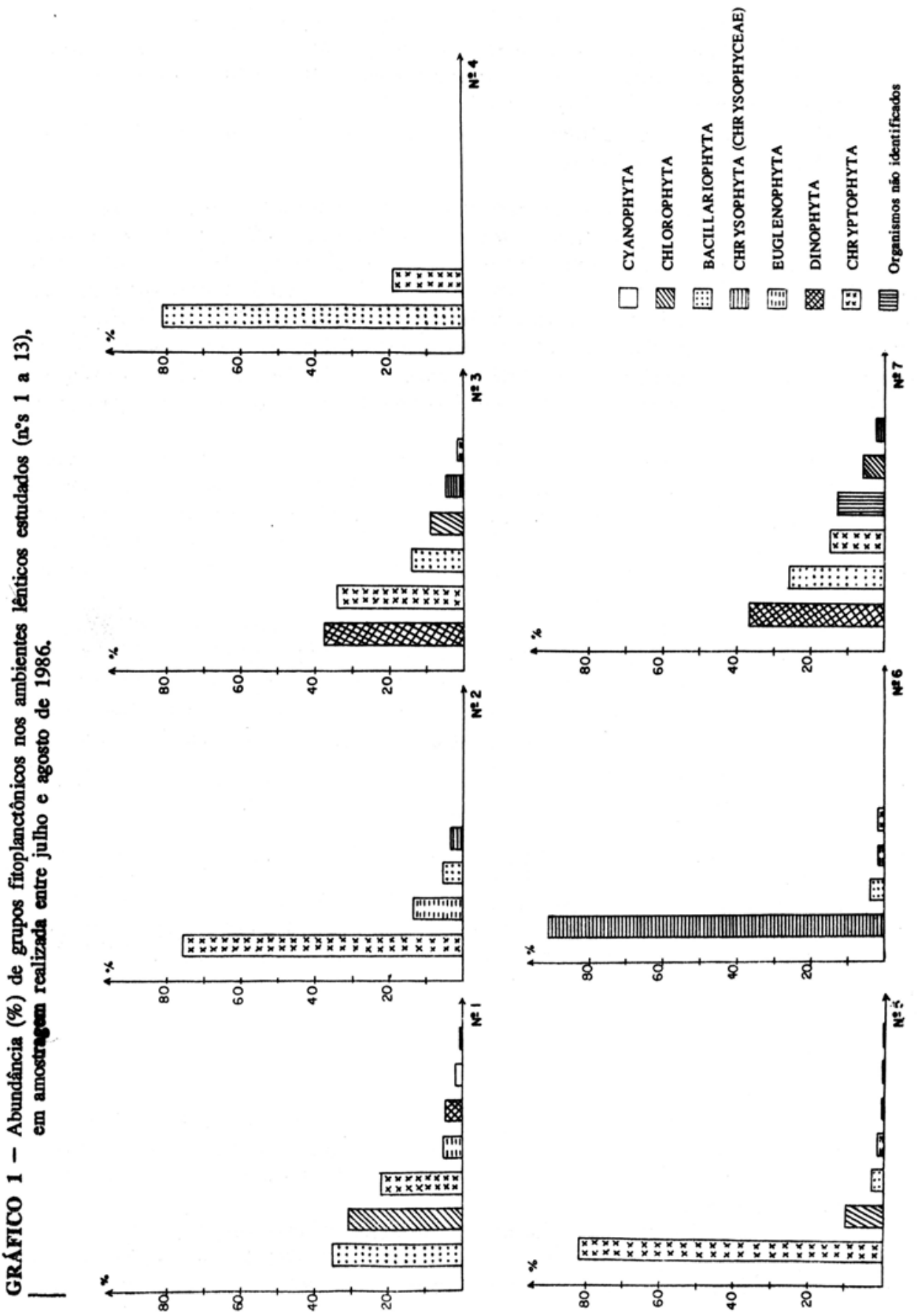




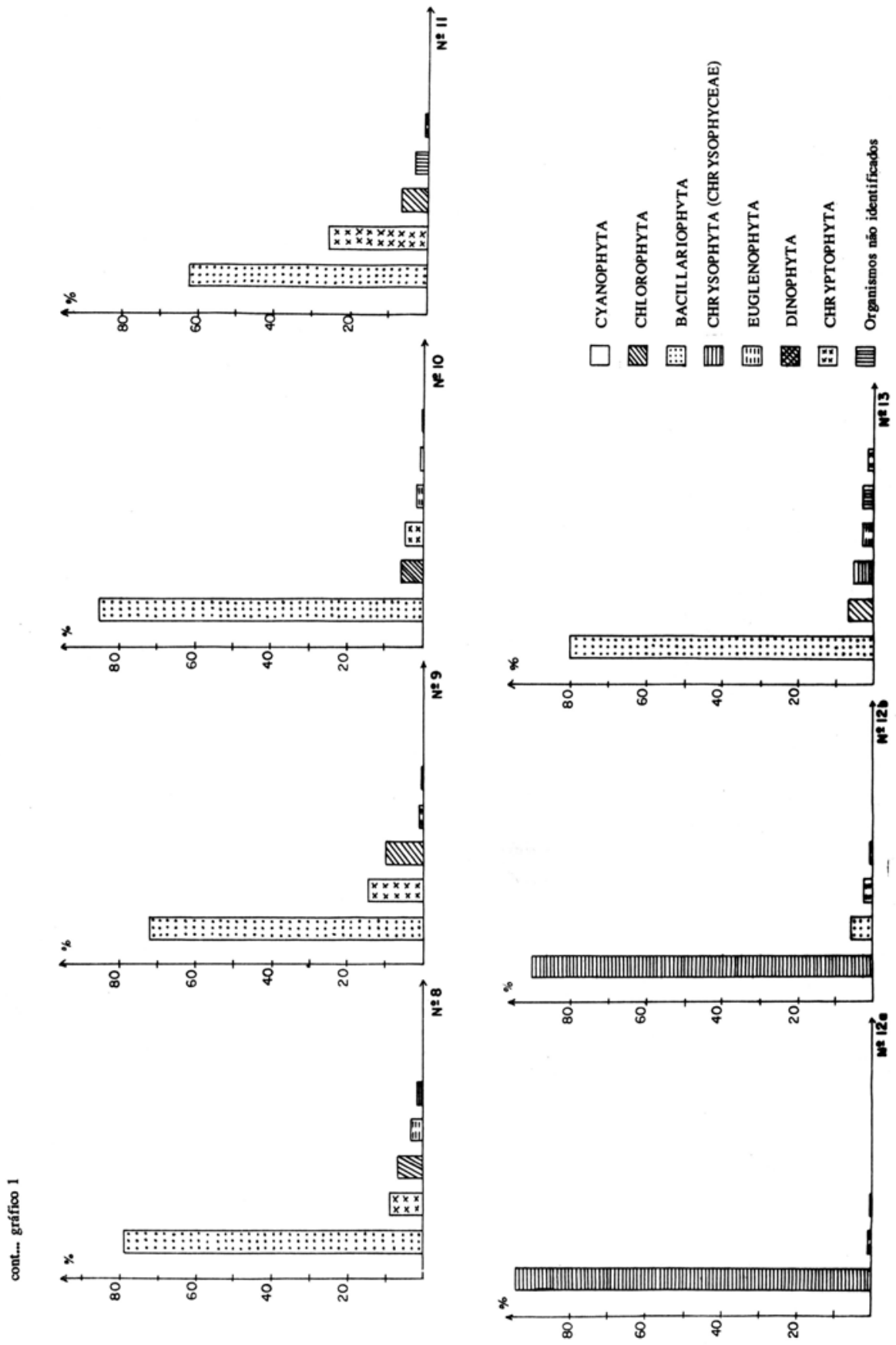


ácido, em ambientes do quadrante sudeste (Mapa $\mathrm{n}^{\circ} 1$; ambientes $\mathrm{n}^{\circ} 1,3$ a 5 e 7 a 11).

2. Açude com densa vegetação arbórea marginal (Eucalyptus sp. e mata natural); cobertura de macrófitos superior a $80 \%$ da superfície total do biótopo, com profundidade de até $2,5 \mathrm{~m}$; transparência alta, $\mathrm{pH}$ ácido, condutividade elétrica relativamente baixa e oxigênio dissolvido baixo, tendo a água coloração amarelada, certamente em decorrência de processo intenso de decomposição de matéria orgânica vegetal. A macrófita predominante foi Scirpus cubensis Kunth var. paraguayensis (Maury) Kükenthal, tendo sido encontradas, também, entre outras, Hypericum cf. punctatum Lamarck, Salvinia sp., Myriophyllum brasiliense Cambessedes, Ludwigia cf. peploides (Kunth) Raven, Scirpus submersus C. Wright, Polygonum sp., Nymphoides indica (Linnaeus) O. Kuntze, Reussia subovata (Seuber) Solms-Laubach, Ricciocarpus natans (Linnaeus) Corda e Eichornia crassipes (Martius) Solms-Laubach, sendo essa vegetação comum à maioria dos açudes da região (Mapa $n^{\circ} 1$; ambiente $n^{\circ} 2$ ).

3. Açude sem vegetação marginal ou com poucas gramíneas; ausência de macrófitos; formado por deposição de cinza de carvão mineral; profundidade inferior a $0,50 \mathrm{~m}, \mathrm{pH}$ fortemente ácido, condutividade elétrica relativamente alta e transparência total (Mapa $n^{\circ} 1$; ambiente $n^{\circ} 6$ ).

4. Saco de rio junto ao continente, em Charqueadas, transformado em depósito de cinza, sendo constituído por um canal profundo, margeado por vegetação arbórea formada predominantemente por sarandis, maricás e ingás. Vegetação de macrófitos pouco densa, representada quase que exclusivamente por Utricularia sp. na superficie sob a forma de massas flutuantes e por Scirpus submersus C. Wright, formando cobertura de fundo; transparência e condutividade altas e $\mathrm{pH}$ ácido (Mapa $\mathrm{n}^{\circ} 1$; Ambiente $\mathrm{n}^{\circ} 12 \mathrm{a}, \mathrm{b}$ ).

5. Saco de rio junto a uma ilha, margeado por vegetação arbórea onde predominam sarandis. Transparência relativamente baixa, $\mathrm{pH}$ levemente ácido e condutividade relativamente baixa (Mapa $n^{\circ} 1$; ambiente $n^{\circ} 13$ ).

Através das análises taxonômicas efetuadas, foram identificados 229 táxons, dos quais 213 a nível específico e infra-específico e 15 a nível genérico. Os mesmos estão relacionados na tabela 1 para seus respectivos ambientes; 99 constituem-se em citações novas de ocorrência no Estado do Rio Grande do Sul estando, entre estas, quatro identificadas somente a nível genérico.

Os grupos que mais se destacaram no fitoplâncton e metafíton, quanto ao número de espécies ocorrentes, foram as Chlorophyta, representado predominantemente pelas Zygnemaphyceae (Desmídias), seguido pelas Bacillariophyta (Diatomáceas), Euglenophyta é Cyanophyta.

Quanto à abundância, destacaram-se, no fitoplâncton, as Bacillariophyta (nos corpos d'água $\mathrm{n}^{\circ} 1,4,7$ a 11 e 13), as Cryptophyta (nos corpos d'água $\mathrm{n}^{\circ} 2$, 3 e 5), as Chrysophyta (nos corpos d'água $\mathrm{n}^{\circ} 6$ e 12), conforme gráfico $\mathrm{n}^{\circ}$ 1. O registro das espécies dominantes e abundantes do fitoplâncton para os sistemas amostrados encontra-se na tabela $n^{\circ} 2$.

A tabela $n^{\circ} 3$ registra $o$ resumo da análise quantitativa do fitoplâncton indicando densidade, riqueza, diversidade e uniformidade de espécies. 
A representação gráfica da variação da diversidade específica obtida para os diferentes sistemas amostrados consta no gráfico $\mathrm{n}^{\circ} 2$.

Os valores de alguns parâmetros abióticos $(\mathrm{pH}$, condutividade, temperatura, $\mathrm{O}_{2}$ e transparência) obtidos por ocasião das coletas encontram-se na tabela n. 4.

GRÁFICO 2 - Diversidade especifica (Hs) do fitoplâncton de ambientes lênticos (açudes e sacos) localizados em Charqueadas e regiōes adjacentes.

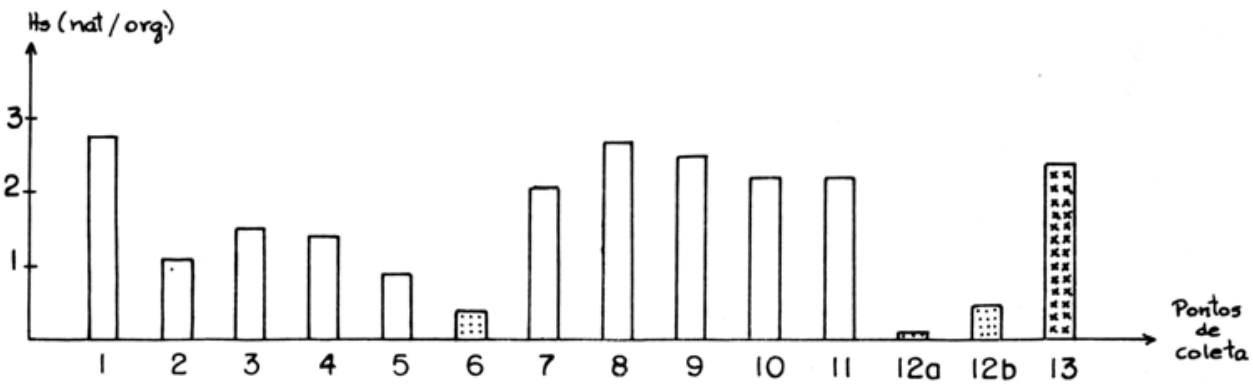

Açudes sem influência direta de depósito de cinza de carvão.

Açudes e sacos do Jacuí, com influência direta de depósito de cinza de carvāo.

Saco do Jacuí sem influência direta de depósito de cinza de carvão. 
TABELA 1 - Presença dos táxons identificados em ambientes lênticos do Município de Charqueadas e adjacências, com base em amostras coletadas em julho e agosto de 1986, com indicação $\left(^{*}\right)$ dos que constituem citaçōes novas de ocorrência para o Estado.

\section{Ambientes amostrados}

$\begin{array}{llllllllllllll}1 & 2 & 3 & 4 & 5 & 6 & 7 & 8 & 9 & 10 & 11 & 12 \mathrm{a} & 12 \mathrm{~b} & 13\end{array}$

Táxons

\section{CYANOPHYTA}

Cyanophyceae

\section{Chroococcales}

Gomphosphaeria lacustris Chodat var. lacustris

Merismopedia glauca (Ehrenberg) Nägeli var.

glauca f. glauca

M. punctata Meyen var. punctata f. punctata

$M$. tenuissima Lemmermann

Microcystis aeruginosa Kützing var. aeruginosa

f. aeruginosa

Ocillatoriales

*Anabaena solitaria Klebahn var. solitaria f. solitaria

*A. subcylindrica Borge

*A. variabilis Kützing ex Bornet \& Flahault

${ }^{*}$ Calothrix brevissima G.S. West var. brevissima f. brevissima

*Oscillatoria granulata Gardner var. granulata

f. granulata

Oscillatoria irrigua Kützing ex Gomont var. irrigua

*O. lacustris (Klebahn) Geitler var. lacustris

$O$. ornata Kützing var. ornata f. ornata

${ }^{*} O$. tenuis C.A. Agardh ex Gomont var. tenuis tenuis t. tenuis

${ }^{*}$ Spirulina princeps West \& West

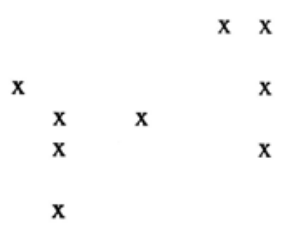

\section{CHRYSOPHYTA}

Chrysophyceae

Chrysomonadales

Dinobryon bavaricum Imhof var. bavaricum $\mathrm{x}$

${ }^{*} D$. divergens Imhof var. divergens

D. sertularia Ehrenberg var. sertularia

Mallomonas sp.

Synura uvella Ehrenberg var. uvella

\section{BACILLARIOPHYTA}

Centrobacillariophyceae

Aulacosira granulata (Ehrenberg) Simonsen var. granulata
A. granulata (Ehrenberg) Simonsen var.

angustissima (Müller) Simonsen

A. italica (Ehrenberg) Ralfs

Cyclotella stelligera Cleve ex Grunow var. stelligera

$\mathrm{x}$

$\mathrm{x}$

$\begin{array}{lll}x & x & x\end{array}$

$\mathrm{x} \mathbf{x}$

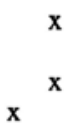

$\mathbf{x}$ 


\section{Táxons}

Achnanthes exigua Grunow var. exigua

A. hungarica Grunow

${ }^{*}$ A. minutissima Kützing var. minutissima

Anomoeoneis exilis Grunow var. exilis

Cymbella affinis Kützing var. affinis

C. amphicephala Nägeli var. amphicephala

C. gracilis (Rabenhorst) Cleve var. gracils

C. microcephala Grunow var. microcephala

C. minuta Hilse ex Rabenhorst

C. turgida (Gregory) Cleve

${ }^{*}$ Eunotia alpina (Nägeli) Hustedt

E. exigua (Brébisson) Grunow var. exigua

$E$. lunaris (Ehrenberg) Grunow var. lunaris

E. pectinalis (Ktzing) Rabenhorst var.

pectinalis

E. pectinalis (Kützing) Rabenhorst var. minor

(Kützing) Rabenhorst

E. pectinalis (Kützing) Rabenhorst var. rostrata Germain

E. praerupta Ehrenberg var. bidens (Ehrenberg)

Grunow

E. tenella (Grunow) Hustedt

Fragilaria sp.

Frustulia rhomboides (Ehrenberg) De Toni var. saxonica (Rabenhorst) De Toni

Gomphonema angustatum (Kützing) Rabenhorst

var. angustatum

G. augur Ehrenberg var. augur

G. gracile Ehrenberg var. gracile

$G$. intricatum Kützing var. intricatum

G. parvulum (Kützing) Grunow var. parvulum

G. parvulum (Kützing) Grunow var. micropus (Kützing) Cleve

Hantzschia amphioxys (Ehrenberg) Grunow

var. amphioxys

Navicula cryptocephala Kützing var.

cryptocephala

N. pupula Kützing var. pupula

Neidium sp.

Nitzschia ignorata Krasske

$N$. hantzschiana Rabenhorst var. hantzschiana

$N$. palea (Kützing) W. Smith var. palea

$N$. parvula Lewis non W. Smith

* Pinnularia acoricola Hustedt var. acoricola

${ }^{*} P$. acuminata W. Smith var. acuminata

$P$. biceps W. Smith

Pinnularia borealis Ehrenberg var. borealis

$P$. braunii (Grunow) Cleve var. amphicephala

(A. Meyer) Hustedt

$P$. divergens $\mathrm{W}$. Smith var. divergens
$\mathbf{X}$

$\mathbf{X}$

$\mathbf{X}$

$\mathbf{x}$

X

$\mathrm{X}$

$\mathrm{x}$

$\mathbf{x}$

$\begin{array}{lll}\mathbf{X} & \mathrm{X} & \mathbf{x}\end{array}$

$\mathrm{x}$

x

$\mathrm{x}$

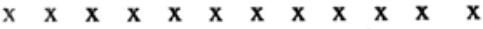

X

X

$\mathbf{x}$

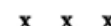

$\mathrm{x} \mathbf{x}$

$\mathbf{x} \mathbf{x}$

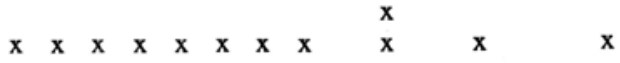
x $x$

X

X

X

$\mathrm{x}$ 
Ambientes amostrados

$\begin{array}{llllllllllllll}1 & 2 & 3 & 4 & 5 & 6 & 7 & 8 & 9 & 10 & 11 & 12 \mathrm{a} & 12 \mathrm{~b} & 13\end{array}$

\section{Táxons}

${ }^{*} P$. divergentissima (Grunow) Cleve var. divergentissima

P. gibba Ehrenberg var. gibba

P. ' maior (Kützing) Cleve var. maior

P. microstauron (Ehrenberg) Cleve var. microstauron

P. obscura Krasske var. obscura

$P$. viridis (Nitszch) Ehrenberg var. viridis

Surirella bisseriata Brébisson var.

bisseriata

$S$. linearis W. Smith var. linearis

Stauroneis phoenicenteron Ehrenberg var. phoenicenteron

* Stenopterobia intermedia Lewis var. intermedia

Synedra acus Kützing var. acus

$S$. rumpens Kützing var. rumpens

\section{CHLOROPHYTA}

Chlorophyceae

Volvocales

Eudorina elegans Ehrenberg

Eudorina unicocca Smith

Pandorina morum (Müller) Bory

Volvox sp.

Chlorococcales

*Ankistrodesmus falcatus (Corda) Ralfs var. falcatus

A. gracilis (Reinch) Korshikow

*A. spiralis (Turner) Lemmermann var. fasciculatus G.M. Smith

Botryococcus braunii Kützing

Coelastrum sphaerium Nägeli

Crucigenia rectangularis (A. Braun) Gay

Dimorphococcus lunatus A. Braun

Golenkinia sp.

Kirchneriella lunaris (Kirchner) Moebius

var. lunaris

*Ophyocytium sp.

Oocystis sp.

Monoruphidium contortum Komarkova-

Legnerova var. contortum

*Pediastrum duplex Meyen var. duplex

$P$. simplex Meyen var. simplex

*Quadrigula sp.

* Scenedesmus acuminatus (Lagerheim) Chodat var. acuminatus

$\mathbf{x}$

$\mathbf{x}$

$\mathbf{x}$

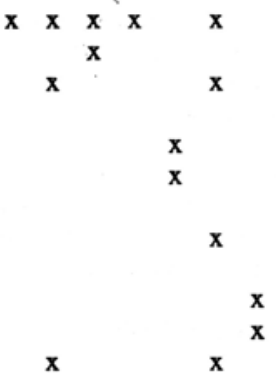

$\mathrm{x}$

$\mathbf{x}$

$\mathrm{x}$

$\mathbf{x}$

$\mathbf{x}$

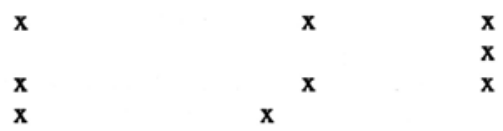

$\begin{array}{lllllllll}\mathbf{x} & \mathbf{x} & \mathbf{x} & \mathbf{x} & \mathbf{x} & & & \mathbf{x} & \\ & & \mathbf{x} & \mathbf{x} & & \mathbf{x} & & \mathbf{x} \\ & & & & \mathbf{x} & & & \\ & & & & & & & & \end{array}$

$\mathbf{x}$

$\mathbf{x}$

$\mathbf{x}$

$\mathbf{x}$

$\mathbf{x}$

$\mathbf{x}$

$\mathbf{x}$

$x$

$\mathbf{x}$

$S$. acutus Meyen var. acutus

* $S$. arcuatus Lemmermann

* S. armatus (Chodat) G.M. Smith var. armatus

* $S$. brasiliensis Bohlin var. brasiliensis 
Ambientes amostrados

Táxons

S. quadricauda (Turpin) Brébisson

${ }^{*}$ Tetrallanthus lagerheimii Teiling

Oedogoniophyceae

Oedogoniales

Bulbochaete sp.

Oedogonium sp.

Zygnemaphyceae

Gonatozygales

Gonatozygon monotaenium De Bary var.

monotaenium

G. pilosum Wolle

Mesotaeniales

*Mesotaenium spp.

Netrium digitus (Ehrenberg) Itzigson \& Roth var. digitus

${ }^{*}$ Spirotaenia condensata Brébisson

Desmidiales

${ }^{*}$ Actinotaenium pyramidatum (West \& West) Teiling

Arthrodesmus mucronulatus Nordstedt var. mucronulatus

${ }^{*}$ A. octocornis (Ehrenberg) Archer var. octocornis

Bambusina brebissonii Kützing var.

brebissonii

${ }^{*}$ Closterium archerianum Cleve

${ }^{*} C$. closterioides (Ralfs) Louis \& Peeters var. closterioides

${ }^{*} C$. cynthia De Notaris var.cynthia

C. ehrenbergii Meneghini var.?

${ }^{*} C$. gracile Brébisson var. intermedium

Irénée-Marie

C. kuetzingii Brébisson var. kuetzingii

C. kuetzingii Brébisson var.?

${ }^{*}$ C. macilentum Brébisson var. macilentum

C. malmei Borge var. malmei

${ }^{*} C$. navicula (Brébisson) Lütkemüller var. crassum (West \& West) Grónblad

C. setaceum Ehrenberg var. setaceum

${ }^{*} C$. subfusiforme Messikommer

${ }^{*} C$. turgidum Ehrenberg var. turgidum

${ }^{*}$ Cosmarium abbreviatum Raciborski var. abbreviatum

${ }^{*} C$. alpestre Roy \& Bisset var.alpestre

${ }^{*} C$. anceps Lundell var. anceps

C. connatuin Brébisson var. connatum

${ }^{*} C$. dentatum Wolle var.?

C. denticulatum Borge var.ovale Grónblad

C. elegantissimum Lundell var. elegantissimum f. minor W. West $\begin{array}{llllllllllllll}1 & 2 & 3 & 4 & 5 & 6 & 7 & 8 & 9 & 10 & 11 & 12 \mathrm{a} & 12 \mathrm{~b} & 13\end{array}$ 
Ambientes amostrados

\section{Táxons}

C. lagoense Nordstedt var. amoebum

Förster \& Eckert

${ }^{*}$ C. longicylindricum Scott \& Grönblad

${ }^{*}$ C. luscum Borge

${ }^{*} C$. monomazum Lundell var. dimazum Krieger

C. ornatum Ralfs

${ }^{*} C$. ornatum Ralfs pseudolagoense Förster

${ }^{*} C$. pseudamoenum Wille var. inornatum

(Joshua) Croasdale

C. pseudoconnatum Nordstedt var.

pseudoconnatum

C. pseudopyramidatum Lundell var.

pseudopyramidatum

C. reniforme (Ralfs) Archer

${ }^{*} C$. trilobulatum Reinsch bioculatum Krieger

*Cylindrocystis brebissonii Meneghini var. brebissonii

Desmidium aptogonum Brébisson varaptogonum

D. baileyi (Ralfs) De Bary var.baileyi f.

tetragonum Nordstedt

*Desmidium cilyndricum Greville ex Nordstedt var.cillyndricum

D. laticeps Nordstedt var.quadrangulare

Nordstedt

D. swartzii (C. Agardh) C. Agardh ex Ralfs var.amblyodon (Itzigson) Rabenhorst

D. swartzii (C. Agardh) C. Agardh' ex Ralfs. var.?

Euastrum ansatum Ehrenberg ex Ralfs var. ansatum

*E. didelta (Turpin) Ralfs var.quadriceps (Nordstedt) Krieger

E. evolutum (Nordstedt) West \& West var. evolutum

E. gemmatum (Brébisson) Ralfs var.gemmatum

${ }^{*} E$. oblongum (Greville) Ralfs var. oblongum

*E. subintegrum Nordstedt var.brasiliense Grönblad

*Groembladia undulata Förster var.undulata Hyalotheca dissiliens (Smith) Brébisson

H. mucosa (Mertens) Ehrenberg

*Micrasterias borgei Krieger var. borgei

M. furcata C. Agardh ex Ralfs var. furcata

$M$. laticeps Nordstedt var. laticeps

${ }^{*} M$. mahabuleshwarensis Hobsen var. mahabuleshwarensis

M. truncata (Corda) Brébisson ex Ralfs var. pusilla G.S. West

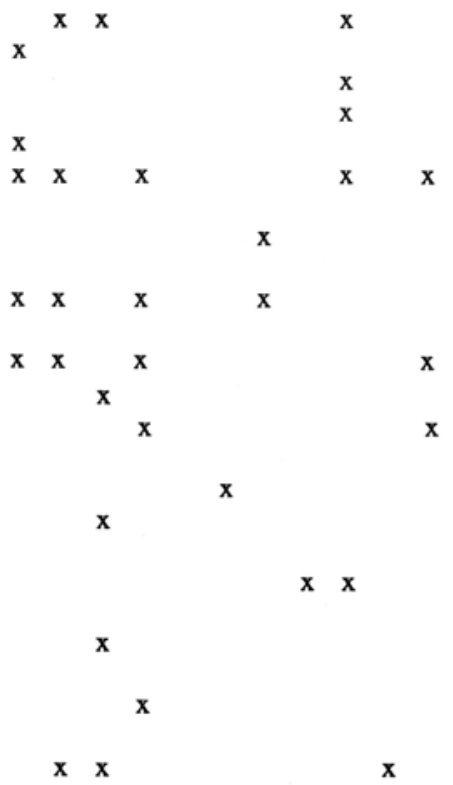

$\mathbf{x}$

$\mathbf{x} \mathbf{x}$

$\mathbf{x}$

$\mathbf{x}$

$\mathbf{x}$

$\mathbf{x}$

$\mathbf{x}$ 
Ambientes amostrados

\section{Táxons}

*Onichonema filiforme (ehrenberg) Roy \& Bisset

$\mathbf{X}$

O. Laeve Nordstedt

* O. uncinatum Wallich var. uncinatum

* Phymatodocis alternans Nordsted

* Pleurotaenium caldense Nordsttedt var caldense

* $P$. caldense Nordstedt var. cristatum (Turner) Krieger

*P. cylindricum (Turner) Schmidle var. stuhlmannii (Hieronymus) Krieger

P. ehrenbergii (Brébisson) De Bary var. ehrenbergii

${ }^{*} P$. minutum (Ralfs) Delponte var. minutum

* $P$. minutum (Ralfs) Delponte var. crassum

(W. West) Krieger

*P. minutum (Ralfs) Delponte var. excavatum Scott \& Grönblad

${ }^{*} P$. ovatum Nordstedt var. obatum

$P$. ovatum Nordstedt var.?

*P. trabecula (Ehrenberg) Nägeli var. trabecula

* Spondylosium pulchellum (Archer) Rabenhorst var. pulchellum

- S. pulchrum Bailey

* Staurastrum bicoronatum Johns. var. simplicius West \& West

* Staurastrum cuspidatum (Brébisson) Ralfs var. alaskanum (Croasdale) Prescott, Bicudo \& Vinyard

* S. dilatatum Ehrenberg var. dilatatum

* S. disputatum West \& West var. sinensis

(Lütkemüller) West \& West

*S. dickiey Ralfs var. circulare Turner

* $S$. gemelliparum Nordstedt

* S. laeve Ralfs

S. leptaeanthum Nordsted var. leptacanthum

$S$. minnesotense Wolle var. minnesotense

S. muticum Brébisson ex Ralfs var. muticum $\mathbf{x}$

* $S$. muticum Brébisson ex Ralfs f. minus

Rabenhorst

S. polymorphum Brébisson var. polymorphum

$S$. quadrangulare Brébisson var. quadrangulare

$S$. rotula Nordstedt var. rotula

S. setigerum Cleve var. pectinatum

West \& West

*Staurodesmus selenaeus (Grönblad) Teiling,

* S. spencerianus (Maskell) Teiling

*Teilingia granulata (Roy \& Bisset) Bourrelly Tetmemorus brebissonii (Meneghini) Ralfs var. brebissonii $\mathbf{x}$

$\mathrm{x}$

$\mathrm{x}$

$\mathbf{x}$

$\mathrm{x}$

$\mathbf{x}$

$\mathbf{x}$

$\mathbf{X}$

$\mathbf{X}$

$\mathrm{x}$

$\mathbf{x}$

X

$\mathbf{x}$

x

$\mathbf{x}$

X

X

$\mathrm{x}$

x

X

X

$\mathbf{x}$

x

x

$$
\text { x }
$$

X

X $\mathrm{X}$

x

$\mathrm{x}$

$\mathrm{x}$

x

x

$\mathrm{x}$

X 
Táxons

*Triploceras gracile Bailey var bidentatum Nordstedt

*Xanthidium calcarato-aculeatum (Hieronymus) Schmidle

Zygnemales

${ }^{*}$ Mougeotia laetevirens (A. Braun) Wittrock Spirogyra sp.

\section{EUGLENOPHYTA}

Euglenophyceae

Euglenales

*Euglena mutabilis Schmitz var. mutabils

"E. oxyuris Schmarda

${ }^{*}$ E. spirogyra Ehrenberg

${ }^{*} E$. velata Klebs

*Lepocinclis cylindrica (Korschkoff) Conrad

*Phacus curvicauda Swirenko var. curvicauda

${ }^{*} P$. longicauda (Ehrenberg) Dujardin var.

longicauda

${ }^{*} P$. rudicula (Playfair) Pochmann

${ }^{*} P$. undulatus (Skvortzov) Pochmann $\mathrm{f}$. multiundulata (Halasz) Huber-Pestalozzi

Trachelomonas armata (Ehrenberg) Stein var. armata

$T$. armata (Ehrenberg) Stein var. longispina

Playfair emend. Deflandre

Trachelomonas hispida (Perty) Stein emend.

Deflandre

${ }^{*} T$. hispida (Petry) Stein emend. Deflandre

var. duplex Deflandre

${ }^{*} T$. klebsii Deflandre

*T. verrucosa Stokes

$T$. volvocina Deflandre var. volvocina

$T$. volvocina Deflandre var. derephora Conrad

$T$. volvocinopsis Swirenko var. volvocinopsis

Peranematales

*Anisonema acinus (Dujardin) Lemmermann

*Entosiphon sulcatum (Dujardin) Stein var. acuminatum Lemmermann

*Gyropaigne sp.

DINOPHYTA

Dinophyceae

Peridiniales

*Peridinium gatunense Nygaard var. gatunense

Chryptophyta

Chryptomonadales

Chrytomonas sp. $\mathrm{x}$

$\mathbf{x}$

$\mathbf{x}$

X $\quad$ X $\quad x \quad x$

$\mathbf{x}$

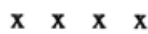

X

X

X $\mathrm{x}$

X

X

X

x $\quad \mathrm{x}$

$\mathrm{X}$

X

x

$\mathrm{x}$

$\begin{array}{ll}\mathbf{x} & \mathbf{x} \\ \mathbf{x} & \end{array}$

X

X

x

$\begin{array}{llll}\mathbf{x} & \mathrm{x} & \mathrm{x}\end{array}$

x $\mathbf{x}$

*Citaçōes novas para o Estado do Rio Grande do Sul. 


\section{Comentários}

É conhecido o fato de que a pirita ( $\mathrm{FeS}$ ), existente em cinza de carvão mineral, é responsável pela acidificação das águas em contacto com esses depósitos. Como processo desencadeador de alterações na água pode ser apontada a oxidação, sendo produtos resultantes das reações que se sucedem sulfitos, sulfatos, óxidos e hidróxidos de ferro, como também ácido sulfúrico, que deter mina valores baixos de $\mathrm{pH}$ na água.

Para os ambientes ácidos amostrados que se constituem em depósito de cinza de carvão mineral ( $n^{\circ} 6$ e 12) e, portanto, sofrem influência direta desses materiais, foram constatados valores de $\mathrm{pH}$ muito baixos, assim como de condutividade e transparência altos (Tab. 4). O oxigênio dissolvido do ambiente $\mathbf{n}^{\circ}$ 6 , entretanto, não apresentou valor baixo conforme o esperado para local com provável processo de oxidação da pirita. Tal fato ocorreu, possivelmente, pela pequena profundidade do açude e conseqüente oxigenação da água por ação dos ventos. Nesses bićtopos, a estrutura das comunidades do fitoplâncton indicou os valores mais baixos para riqueza de espécies e diversidade, o que se refletiu na homogeneidade de distribuição dos organismos ocorrentes (Tab. 3); foram dominantes organismos da classe Chrysophyceac não identificados. $O$ bentos e o metafiton constituiram-se de comunidades formadas basicamente por espécies acidófilas, mas a flora de desmídias (Chlorophyta - Zygnemaphyceae) mostrouse muito pobre ou inexistente. Tal fato confirmaria a observação referida por COESEL et alii (1978) de que existiria uma relação entre a redução da flora desse grupo de organismos e a acidificação de ambientes aquáticos oriunda da lixiviação de solos acidificados por resíduos industriais.

Considerando a influência indireta - acidificação provocada por efluentes aéreos - pode ser observada, no gráfico $\mathrm{n}^{\circ} 2$, uma tendência na redução dos índices de diversidade dos açudes mais distantes da área industrial de Charqueadas aos mais próximos, no quadrante sudeste (corpos d'água de $\mathrm{n}^{\circ} 1$ a 5 ) e uma diferença evidente entre os valores dos quadrantes sudeste e sudoeste. Esta região constitui-se em área onde, teoricamente, haveria maior influência da poluição atmosférica, pelo fato ce localizar-se no quadrante da direção predominante dos ventos. Saliente-se o fato de que o açude $n^{\circ} 2$ não pode constituir padräo de comparação, visto ser ambiente acidificado naturalmente devido à degradação de matéria orgânica proveniente da densa cobertura de macrófitos. A acidificação dos solos do quadrante, fato que seria esperado em decorrência da poluição industrial aérea existente, não foi confirmada por medições; entretanto, a ocorrência constatada de manchas de Euglena mutabilis Schmitz na superfície de solos úmidos confirmaria a existência de solos ácidos naquele quadrante, visto ser a espécie organismo tipicamente acidófilo.

Quanto à dominância no fitoplâncton foram encontradas espécies nessa condição somente para os corpos d'água mais fortemente ácidos (n ${ }^{\circ} 2,6$ e 12); os demais apresentaram diferentes espécies apenas em condiçõs de abundância, o que poderia refletir maior estabilidade (Tab. 2). 
TABELA 2 - Táxons dominantes $(\underline{X})$ e abundantes $(X)$ do fitoplâncton em corpos d'água lênticos estudados (N.s 1 a 13), com base em amostras coletadas entre julho e agosto de 1986.

\section{Ambientes amostrados}

$\begin{array}{llllllllllllll}1 & 2 & 3 & 4 & 5 & 6 & 7 & 8 & 9 & 10 & 11 & 12 \mathrm{a} & 12 \mathrm{~b} & 13\end{array}$

\section{Táxons}

\section{Achnanthes hungarica}

Ankistrodesmus gracilis

Anomoeoneis exilis

Aulacosira granulata

A. granulata var. angustissima

A. granulata var. angustissima f. curvata

Chyptomonas sp.

Chryptophyceae não-identificada

Chrysophyceae não-identificada

Cymbella affinis

C. minuta

Euastrum sp.

Euglena sp.

Eunotia alpina

E. pectinalis

$E$. pectinalis var. minor

Frustulia rhomboides var. saxonica

Gomphonema gracile

G. parvulum

G. parvulum var. micropus

Navicula cryptocephala

Navicula sp.

Nit zschia palea

N. parvula

Peridinium gatunense

Peridinium sp.

Pinnularia acoricola

$P$. microstauron

Scenedesmus armatus

Stenopterobia intermedia

Synedra acus

Organismos não-identificados

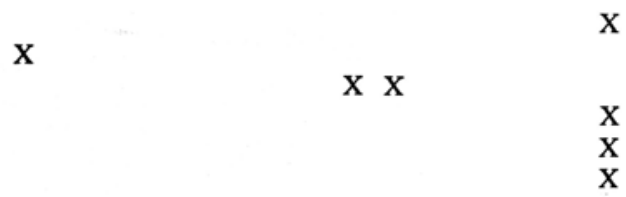

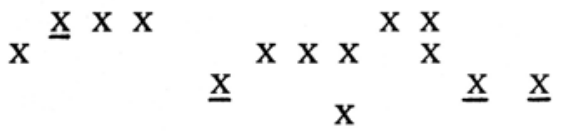

$\mathrm{X}$

$\mathbf{X}$

X

\section{$\mathrm{X}$}

$\mathrm{X}$

$\mathrm{X}$
$\mathrm{X}$
$\mathbf{X}$

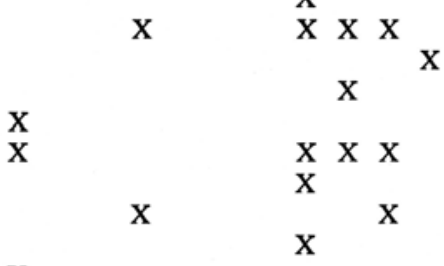

X

$\begin{array}{lllll} & \mathrm{X} & & \mathrm{X} & \\ & & & & \mathrm{X} \\ \mathrm{X} & & \mathrm{X}^{\mathrm{X}} & & \end{array}$

$$
\begin{aligned}
& \mathrm{X}^{\mathrm{X}} \mathrm{X} \\
& \mathrm{X}
\end{aligned}
$$<smiles>C1CCC1</smiles> 
tal representatividade não foi evidenciada, senǎo, então, as Chrysophyceae as que apresentaram maior destaque.

Quanto à composição das comunidades, verificou-se que para os ambientes com rejeito de carvão os táxons que atingiram maior densidade constituem-se em espécies referidas na literatura como acidófilas, tolerantes a flutuações de $\mathrm{pH}$ e à deficiência periódica de oxigênio, comó também oligotróficas, capazes de viver em águas com baixa concentração de nutrientes (SCHOEMAN, 1973; FOGED, 1976; HUSTEDT, 1957; GERMAIN, 1981; SLADECEK, 1973; CHOLNOKY, 1968 e JÖRGENSEN, 1948). Dentre elas, podem ser destacadas as diatomáceas Pinnularia microstauron (Ehrenberg) Cleve e $P$. gibba Ehrenberg encontradas predominantemente no bentos. Associada àquelas e destacando-se quanto à densidade, ocorreu Euglena mutabilis Schmitz, também referida na literatura para pH ácido e ambiente oligotrófico. HEIN (1959) registra a espécie para águas usadas, fortemente ácidas - pH 1,9 - oriundas de uma fábrica de ácido sulfúrico e LACKEY (1939) refere-a dominante em águas residuárias de uma mina de carvão, bastante ácidas - pH de 1,8 a 3,9.

No metafiton, junto a emaranhados sobrenadantes de Utricularia sp., e em material de fundo junto a Scirpus submersus C. Wright em Saco do Jacuí (ambiente $\mathrm{n}^{\circ} 12$ ), que se constitui em depósito de cinza de carvão, destacou-se, em densidade, a espécie Eunotia exigua (Brébisson) Grunow referida por SCHOEMAN (1973) como amplamente distribuída em águas ácidas, oligotróficas e ricas em oxigênio. Essa espécie foi encontrada também no açude $n^{\circ} 6$, mas com menor representatividade quanto à densidade.

$\mathrm{Na}$ composição das comunidades fitoplanctônicas dos demais ambientes amostrados, podemos observar, entre as espécies abundantes (Tab. 2), a presença. de representantes de algas referidas na literatura como sendo de ambientes ácidos ou tolerantes a amplas flutuações de $\mathrm{pH}$. Entre estas, podem ser apontadas: Pinnularia microstauron (Ehrenberg) Cleve, também referida como indicadora de despejos industriais ricos em ferro (PALMER, 1959); Frustulia rhomboides (Ehrenberg) De Toni var. saxonica (Rabenhorst) De Toni, referida por SCHOEMAN (1973) como espécie de ambientes ácidos e pH ótimo em torno de 6, por ROUND (1962) como tendo bom desevolvimento junto â região de descargas de sulfito, provenientes de despejos industriais e por CHOLNOKY (1968) para águas com grande quantidade de ácido sulfúrico proveniente de zona de mineração de ouro; Gomphonema parvulum (Kützing) Grunow, capaz de tolerar amplas flutuaçōes de $\mathrm{pH}$, sendo encontrada também em águas ácidas e ambientes eutróficos (SCHOEMAN, 1973) e em águas com limites de $\mathrm{pH}$ variando de 2 a 6,5 (SCHOEMAN, 1973; FOGED, 1979); Gomphomema gracile Ehrenberg, referida por GERMAIN (1981) como espécie comum em águas ácidas; Pinnularia acoricola Hustedt, referida por SCHOEMAN (1973) para pH ótimo em tomo de 5 e tolerante à deficiência de oxigênio; Anomoeoneis exilis Grunow, indicada por alguns autores para águas levemente ácidas ou levemente alcalinas, mas por JORGENSEN (1948) para pH indiferente; e Eunotia pectinalis (Kützing) Rabenhorst var. minor (Kützing) Rabenhorst, indicada para $\mathrm{pH}$ entre 4 e 7,5 (FOGED, 1977), sendo capaz de tolerar flutuações apesar de ter pH ótimo em torno de 6,5, segundo SCHOEMAN (1973) e sendo considerada acidófila por JÖRGENSEN (1948) e HUSTEDT (1957). 
TABELA 3 - Resumo da análise quantitativa do fitoplâncton de corpos d'água lênticos estudados ( $\mathrm{n}^{\circ} 1$ a 13), com base em estudo de amostras coletadas entre julho e agosto de 1986.

\begin{tabular}{lcccc}
\hline $\begin{array}{l}\text { Corpos } \\
\text { d'água }\end{array}$ & $\begin{array}{c}\text { densidade } \\
\text { total } \\
\left(\mathrm{n}^{\circ} \text { org/ml) }\right.\end{array}$ & $\begin{array}{c}\text { riqueza } \\
\text { de } \\
\text { espécies }\end{array}$ & $\begin{array}{c}\text { diversidade } \\
\text { espcífica } \\
\text { (nat/org) }\end{array}$ & $\begin{array}{c}\text { uniformidade } \\
\text { de } \\
\text { espécies }(\%)\end{array}$ \\
\hline $\mathrm{n}^{\circ} 1$ & 16.085 & 37 & 2,775 & 76,85 \\
$\mathrm{n}^{\circ} 2$ & 16.405 & 13 & 1,123 & 43,78 \\
$\mathrm{n}^{\circ} 3$ & 3.259 & 14 & 1,436 & 54,41 \\
$\mathrm{n}^{\circ} 4$ & 1.069 & 9 & 1,329 & 60,48 \\
$\mathrm{n}^{\circ} 5$ & 96.687 & 53 & 0,899 & 25,71 \\
$\mathrm{n}^{\circ} 6$ & 83.509 & 14 & 0,424 & 26,34 \\
$\mathrm{n}^{\circ} 7$ & 2.342 & 20 & 2,080 & 78,81 \\
$\mathrm{n}^{\circ} 8$ & 2.902 & 33 & 2,678 & 89,39 \\
$\mathrm{n}^{\circ} 9$ & 15.378 & 15 & 2,587 & 63,12 \\
$\mathrm{n}^{\circ} 10$ & 46.804 & 3 & 2,207 & 82,23 \\
$\mathrm{n}^{\circ} 11$ & 5.703 & 6 & 2,227 & 10,64 \\
$\mathrm{n}^{\circ} 12 \mathrm{a}$ & 9.493 & 20 & 0,117 & 25,56 \\
$\mathrm{n}^{\circ} 12 \mathrm{~b}$ & 8.251 & & 0,458 & 79,58 \\
$\mathrm{n}^{\circ} 13$ & 1.344 & & 2,384 & \\
\hline
\end{tabular}

TABELA 4 - Parâmetros abióticos medidos em sete ambientes amostrados na região de Charqueadas, no período de julho/agosto de 1986 .

\begin{tabular}{|c|c|c|c|c|c|}
\hline Dados abióticos & $\mathrm{pH}$ & $\begin{array}{l}\text { Condutividade } \\
\text { (umhos) }\end{array}$ & $\begin{array}{c}\text { Temperatura } \\
\left({ }^{\circ} \mathrm{C}\right)\end{array}$ & $\begin{array}{c}\mathrm{O}_{2} \\
(\mathrm{mg} / \mathrm{l})\end{array}$ & $\begin{array}{c}\text { Transparência } \\
(\mathrm{cm})\end{array}$ \\
\hline $\begin{array}{l}\text { Açude no Horto Florestal } \\
\text { da CEE Guaiba }\left(n^{\circ} 2\right)\end{array}$ & 4,6 & 18 & 15,2 & 3,0 & 100 \\
\hline $\begin{array}{l}\text { Açude no Horto Florestal } \\
\text { da CEE Guaiba (n' 3) }\end{array}$ & 6,0 & 20 & 16,0 & 4,5 & - \\
\hline Açude da Granja Carola (n० 4) & 5,6 & 32 & 17,0 & 8,2 & - \\
\hline Açude Passo Sete $\left(\mathrm{n}^{\circ} 5\right)$ & 6,8 & 30 & 16,0 & - & - \\
\hline $\begin{array}{l}\text { Açude de rejeito de carvão- } \\
\text { Charqueadas }\left(n^{\circ} 6\right)\end{array}$ & 2,8 & 800 & 16,0 & 8,9 & total \\
\hline $\begin{array}{l}\text { Saco do Rio Jacuí cl } \\
\text { rejeito de carvaao }\left(\mathrm{n}^{\circ} 12\right)\end{array}$ & $4,0^{*}$ & alta* & - & - & 200 \\
\hline $\begin{array}{l}\text { Saco da Ilha dos Dorneles- } \\
\text { Rio Jacuí }\left(n^{\circ} 13\right)\end{array}$ & 6,2 & 40 & 15,0 & 8,0 & 45 \\
\hline
\end{tabular}

- medições não-efetuadas.

*dados obtidos com base em mediçōes realizadas por equipe responsável por avaliação abiótica (Epstein, informação verbal). 
Entre as espécies abundantes encontradas estão registradas também Gomphonema parvulum (Kützing) Grunow, Navicula cryptocephala Kützing e Nitzschia palea (Kützing) W. Smith, que são consideradas, por autores diversos, indicadoras de poluição de origem orgânica. A primeira é referida por SCHOEMAN (1973) como tolerante a condições eutróficas, sendo registrada por SLÁDECEK (1973) como indicadora de ambiente alfa-mesossapróbico. A segunda é registrada por SLÁDECEK (1973) para ambiente predominantemente beta-mesossopróbico. A última é referida por vários autores como muito difundida em diferentes meios, por suportar alterações diversas, com grau de poluição acentuado; EVANS (1958) refere-a como bom indicador de condições eutróficas quando ocorre em grande número, sendo reconhecida por SLÁDECECK (1973) e por vários autores, como de ambiente alfa-mesossapróbico; é referida por MARGALEF (1969) como resistente a altas concentrações de cobre e cromo; e por PALMER (1959) como indicadora de altas concentrações de cobre, ácido sulfúrico e cromo. Também Synedra acus Kützing, Cymbella minuta Hilse ex Rabenhorst e Nitzschia parvula Lewis non W. Smith estão classificadas no sistema de sapróbios de SLÁDECEK (1973) como organismos de ambientes beta-mesossopróbicos.

Considerando indicadores para mesossaprobidade, podem ser observadas, entre as espécies ocorrentes (Tab. I), várias indicadoras desse tipo de ambiente. Entretanto, não foi observada dominância de qualquer uma delas e a densidade relativa das mesmas foi baixa, a não ser no açude $n^{\circ} 4$, da Fazenda Carola, onde Nitzschia palea (Kützing) W. Smith foi abundante tendo, então, os valores mais altos para densidade relativa observados para a espécie (305 org./ml). Poderia esse ambiente estar sujeito a uma sobrecarga maior de origem orgânica e a mesma estar interferindo, também, nos baixos níveis de riqueza de espécies, densidade e diversidade específica encontrados. Entretanto, das três espécies que foram abundantes para aquele corpo d'água, a terceira que se sobressaiu em número - Frustulia rhomboides (Ehrenberg) De Toni var. saxonica (Rabenhorst) De Toni - constitui-se também em organismo acidófilo, já referido anteriormente, o que indicaria condições ácidas para o local. Em outros açudes, como os de $n^{\circ} 1,8,9$, e 10 , também se fizeram representar como abundantes espécies indicadoras de mesossaprobidade, não tendo, entretanto, sido observada redução nos valores obtidos para riqueza de espécies e diversidade.

Quanto a presença, Criptomonas sp., Frustulia rhomboides (Ehrenberg) De Toni var. saxonia (Raberhorst) De Toni e Nitzschia palea (Kützing) W. Smith foram os táxons que mais se destacaram, por ocorrerem na maioria dos sistemas amostrados.

\section{Conclusōes}

Ficaram evidenciadas diferenças estruturais entre as comunidades de algas de ambientes lênticos ácidos (sujeitos à influência direta de cinza de carvão mineral) e aquelas de ambientes aparıntemente em condiçöes naturais. $O$ índice de diversidade do fitoplâncton desses ambientes fortemente ácidos foi relativa- 
mente baixo, assim como a riqueza de espécies, sendo que a composição taxonômica do metafiton e bentos teve, como predominantes, organismos referidos na literatura como tipicamente acidófilos, oligotróficos e capazes de sofrer deficiências periódicas de oxigênio.

Essas diferenças, certamente, constituem-se em reflexo de alterações ambientais extremas, tais como $\mathrm{pH}$ acentuadamente ácido, condutividade elevada e, talvez, baixa disponibilidade de nutrientes que, entre outros fatores, teriam sido limitantes ao desenvolvimento da maioria dos táxons componentes da ficoflora da região.

A possível acidificação de açudes por aporte de poluentes aéreos - provenientes de chuva ácida ou de lixiviação de solos acidificados - foi sugerida, no presente trabalho, para açudes localizados no quadrante sudeste, que sofrem mais intensamente a ação de ventos contendo resíduos de queima do carvão mineral. Tal suposição baseou-se na tendência observada de redução dos índices de diversidade dos açudes mais distantes da área industrial de Charqueadas aos mais próximos, naquele quadrante. Também foi evidente um patamar mais alto para o mesmo índice em açudes dos demais quadrantes, não sujeitos à ação predominante dos ventos na região.

Entretanto, a riqueza de espécies de desmídias, que poderia se constituir em possível indicador de condições de impacto, não apresentou redução considerável em ambientes do quadrante sudeste, se comparada com os demais biótopos estudados. Segundo COESEL et alii (1978), teria sido observada redução da flora desse grupo associada a alteraçōes ambientais introduzidas por efluentes aéreos industriais responsáveis pela acidificação de lagos na Holanda. Com base na constatação feita pelos referidos autores, poderíamos supor que, na maioria dos ambientes analisados no presente trabalho, as alteraçōes ambientais mantenham-se ainda em níveis amenos, não se encontrando o sistema em condições críticas. Contudo, seriam convenientes estudos abrangendo amostragens mais representativas, obtidas ao longo do tempo, como também medições de maior número de variáveis físicas e químicas, que possibilitassem comparações efetivas entre os diferentes sistemas sujeitos à maior ou menor influência de efluentes aéreos industriais.

\section{Agradecimentos}

Às pesquisadoras da Fundação Zoobotânica do Rio Grande do Sul Maria de Lourdes Abruzzi de Oliveira e Marcia Terezinha Menna Barreto das Neves pela identificação de espécies de macrófitos. Ao Dr. Carlos Eduardo de Mattos Bicudo, do Instituto de Botânica da Secretaria do Meio Ambiente do Estado de São Paulo, pela identificação de Cylindrocystis brebissonii Meneghini var. brebissonii. 


\section{Referências bibliográficas}

CHOLNOKY, B.J. 1968. Die Ökologie der Diatomeen in Binnengewässern. apud SCHOEMAN, F.R. 1973. A systematical and ecological study of the diatom flora of Lesotho with special reference to the water quality. Pretoria, V\&R Printers. p.91; 215.

COESEL, P.M.; KWAKKESTEIN, R.; VERSCHOOR, A. 1978. Oligotrophication and Eutrophication some dutch moorland pools, as reflected in theier desmid flora. Hydrobiol., Den Haag, 61 (1): 21-31.

EVANS, J.H. 1958. The survival of freshwater algae during dry periods. Part. I. An investigation of the algae of five small ponds. J. Ecol., London, 46:149-67.

FOGED, N. 1976. Freshwater datoms in Sri Lanka (Ceylon). Vaduz, J. Cramer. (Biblioteca Phycologica, 23)

FOGED, N. 1977. Freshwater Diatoms in Ireland. Vaduz, J. Cramer. (Biblioteca Phycologia, 34)

GERMAIN, H. 1981. Flore des diatomées: eaux douces et saumâtres du Massif Armoricain et des contrées voisines d'Europe occidentale. Paris, Sociéte Nouvelle des Editions Boubé. . 444p., 169est., 2125fig.

HEIN, G. 1953. Ûber Euglena mutabilis und ihr Verhalten zu sauren Medien. In: HUBER-PESTALOZZI, G. 1955. Des Phytoplankton des Süsswassers; Systematik und Biologie. - Euglenophyceen. Stuttgart, E. Schweizerbart'sche. Te.4, p.77-8. (Die Binnengewässer. Ed. August Thienemann, 16)

HUBER-PESTALOZZI, G. 1955. Euglenophyceen. In:

. Das Phytoplankton des Süsswassers; Systematic und Biologie. Stuttgart, E. Schweizerbart'sche. Te.4, 606p., il. (Die Binnengewässer. Ed. August Thienemann, 16)

HUSTED, F. 1957. Die Diatomeenflora des Flussystems der Weser im Geleit der Hausestadt Bremen. Abh. Nat. Ver., Bremen, $34: 181-440$.

JÖRGENSEN, E.G. 1948. Diatom communities in some Danish lakes and ponds. Det Kongelige Danske Vividenskabernes Selskale. apud SCHOEMAN, F.R. 1973. A systematical and ecological study of the diatom flora of Lesotho with special reference to the water quality. Pretoria, V\&R Printers. p.78; 207; 220.

LACKEY, J.B. 1939. Aquatic life in waters polluted by acid mine waste. U.S. Publ. Healter Rep., $54: 740-46$.

LOBO, E. \& LEIGHTON, G. 1986. Estructuras comunitarias de las fitocenosis planctonicas de los sistemas de desembocaduras de rios y esteros de la zona central de Chile. Rev. Biol. Mar., Valparaíso, 22 (1): 1-29.

MARGALEF, R. 1969. El concepto de polucion en Limnologia y sus indicadores biológicos. Suplemento Científico de Agua, Barcelona (7): 105-33.

. 1974. Ecología. Barcelona: Editorial Omega. 768p.

MÜLLER-MELCHERS, F.C. \& FERRANDO, H.J. 1956. Tecnica para el estudio de las diatomeas. Bolm. Inst. Ocean., São Paulo, 7 (1-2): 151-60, jun-dez.

NIESSEN, H. 1956. Ökologische Untersuchungen über die Diatomeen und Desmidiaceen des Murnauer Moores. Archiv. Hydrobiol., Stuttgart, $51: 281-375$.

PALMER, M.C. 1959. Algae in water supplies. Washington, U.S. Department of Health, Education, and Welfare, Public. Heatter Service. 88p., 6 est., 55fig.

PIELOU, E.C. 1975. Ecological diversity. New York, John Wiley. 165p.

RIO GRANDE DO SUL. Secretaria da Agricultura. Departamento de Pesquisa. 1968-1985. Observaçōes meteorológicas; boletim. Porto Alegre, lcópia xerografadal

ROUND, F.E. 1962. The aplication of diatom ecology to water pollution and purification. (Environmental requeriments of plankton alge and their effects on water quality) apud.

SCHOEMAN, F.R. 1973. A systematical and ecological study of the diatom flora of Lesotho with special reference to the water quality. Pretoria, V\&R Printers. p.91.

SCHOEMAN, F.R. 1973. The biology of the algae. 2.ed. London, Eduard Amold Publ. 277p., il.

SCHOEMAN, F.R. 1981. The ecology of algae. 1: ed., Cambridge, Cambridge University Press. 651p., il. 
SCHOEMAN, F.R. 1973. A systematical and ecological study of the diatom flora of Lesotho with spcial reference to the water quality. Pretoria, V\&R Printers. 355p., il.

SHANNON, C.E. \& WEAVER, W. 1949. The mathematical theory of comunication. In: ODUM, E.P. 19. Ecologia. 3.ed. Mexico, Nueva Editorial Interamericana. 639p.

SLÁDECEK, V. 1973. System of water Quality from the Biological Point of View. Arch. Hydrobiol., Stuttgart, $7: 1-218$.

UTHERMÖHL, H. 1958. Zur vervollkommung der quantitativem Phytoplankton-Methdik. Verh. int. Verein. theor. angew. Limnol., Stuttgart, $9: 1-38$, il. 